\title{
Comparaison d'ensilages de maïs de quatre variétés différentes dans l'engraissement du taurillon
}

\author{
A Clinquart, M Gielen, I Dufrasne, L Istasse, \\ C Van Eenaeme, JM Bienfait \\ Université de Liège, faculté de médecine vétérinaire, service de nutrition animale, \\ rue des Vétérinaires, 45, 1070 Bruxelles, Belgique
}

\begin{abstract}
Summary - Comparison of maize silages from 4 varieties in the fattening of young bulis. Relationships were found between chemical composition, digestibility and animal performances for 4 varieties of maize silage offered to growing fattening bulls.
\end{abstract}

Les caractéristiques phytotechniques de différentes variétés de maïs sont connues. Par contre, il n'existe que peu de données comparatives sur les performances de taureaux à l'engrais recevant une ration à base d'ensilage de différentes variétés de maïs.

Matériel et méthodes - Quatre variétés de maïs (Ascot, Gracia, Julius et Lixis) ont été comparées au cours de 2 années chez des taurillons de race Blanc Bleu Belge à l'engrais, d'un poids initial de $350 \mathrm{~kg}$. La $1^{\text {re }}$ année, les 24 animaux étaient de type mixte tandis que la $2^{e}$ année, 12 étaient de type mixte et 12 de type culard. Ils recevaient la même quantité de matière sèche (MS) d'ensilage, qui représentait les $2 / 3$ de la ration. Du tourteau de soja $(1,4 \mathrm{~kg})$ et des pulpes séchées étaient distribuées en complément. Les quantités étaient ajustées tous les 15 $\mathrm{j}$ en vue d'un rationnement proche de ad libitum. Les aliments étaient pesés quotidiennement et distribués individuellement en 2 repass. La digestibilité des rations a été déterminée par collecte des matières fécales pendant 1 semaine. La digestibilité de l'ensilage de maîs a été estimée par soustraction à partir de la digestibilité des autres composants. La digestibilité in vitro du maïs a également été estimée par infra-analyseur. Les animaux ont été pesés tous les $28 \mathrm{j}$. Après abattage en fonction de l'état de finition, la composition des carcasses a été estimée à partir de celle du $7^{\mathbb{E}}$ segment monocostal obtenue par dissection.
Résultats et discussion - Les épis représentaient environ $40 \%$ de la MS de la plante en $1^{\text {re }}$ année et $50 \%$ en $2^{\theta}$ année (tableau I). Les teneurs en MS de l'ensilage de maïs ont été différentes d'une variété à l'autre et, en moyenne, supérieure de plus de $4 \%$ lors de la $2^{\mathrm{e}}$ année. Par contre, il y a eu peu de variation entre variétés en ce qui concerne les teneurs en fibre brute (FB) et protéines brutes (PB). Bien que les différences ne soient pas significatives, la digestibilité de la MS des variétés Julius et Lixis a été plus élevée d'environ $3 \%$ que celle des variétés Ascot et Gracia. La digestibilité de la MS a été supérieure en $2^{\mathrm{e}}$ année alors que celle de la FB a été inférieure. C'est avec la variété Julius que la digestibilité de la FB a été la plus élevée. Les digestibilités moyennes pour les 2 années de $75,3 \%$ et de $67,3 \%$ pour la MS et la FB de Lixis sont comparables aux valeurs rapportées par Gielen et al (1988). La technique d'estimation in vitro n'a pas donné des coefficients identiques aux données in vivo mais a permis d'établir un classement semblable des variétés. Les animaux ont été abattus à un poids proche de $550 \mathrm{~kg}$, ce qui correspondait à une durée d'essai d'environ $160 \mathrm{j}$. II n'y a eu aucun effet significatif des variétés 
Tableau I. Caractéristiques de l'ensilage de maïs et performances des animaux.

\begin{tabular}{|c|c|c|c|c|c|}
\hline Variété de maïs & $A T$ & Ascot & Gracia & Julius & Lixis \\
\hline Proportion d'épis (\%) & $\begin{array}{l}1 \\
2\end{array}$ & $\begin{array}{l}40,2 \\
53,5\end{array}$ & $\begin{array}{l}39,7 \\
44,3\end{array}$ & $\begin{array}{l}44,6 \\
53,1\end{array}$ & $\begin{array}{l}42,4 \\
60,0\end{array}$ \\
\hline $\begin{array}{c}\text { Composition chimique } \\
\text { MS (\%) } \\
\text { PB (\%MS) } \\
\text { FB (\%MS) }\end{array}$ & $\begin{array}{l}1 \\
2 \\
1 \\
2 \\
1 \\
2\end{array}$ & $\begin{array}{r}26,1 \pm 1,4 \\
32,8 \pm 1,4 \\
8,2 \pm 0,6 \\
7,1 \pm 0,2 \\
25,4 \pm 2,5 \\
19,8 \pm 0,7\end{array}$ & $\begin{array}{r}25,5 \pm 0,6 \\
31,2 \pm 1,3 \\
8,4 \pm 0,4 \\
7,2 \pm 0,6 \\
25,7 \pm 3,0 \\
20,7 \pm 1,2\end{array}$ & $\begin{array}{r}27,3 \pm 0,7 \\
32,1 \pm 1,4 \\
8,3 \pm 0,5 \\
7,0 \pm 0,3 \\
25,4 \pm 2,8 \\
20,3 \pm 0,9\end{array}$ & $\begin{array}{r}30,1 \pm 0,3 \\
30,7 \pm 2,1 \\
7,4 \pm 0,5 \\
6,7 \pm 0,4 \\
23,1 \pm 7,8 \\
20,5 \pm 1,1\end{array}$ \\
\hline $\begin{array}{c}\text { Digestibilité in vivo (\%) } \\
\text { MS } \\
\text { FB }\end{array}$ & $\begin{array}{l}1 \\
2 \\
1 \\
2\end{array}$ & $\begin{array}{l}71,5 \pm 2,1 \\
73,3 \pm 2,9 \\
65,4 \pm 0,0 \\
63,4 \pm 0,0\end{array}$ & $\begin{array}{l}72,8 \pm 0,8 \\
72,8 \pm 1,8 \\
67,6 \pm 0,0 \\
63,9 \pm \theta, 0\end{array}$ & $\begin{array}{l}75,0 \pm 1,5 \\
76,3 \pm 2,4 \\
71,7 \pm 0,0 \\
69,0 \pm 0,0\end{array}$ & $\begin{array}{l}74,7 \pm 2,9 \\
75,8 \pm 3,1 \\
67,7 \pm 0,0 \\
66,9 \pm 0,0\end{array}$ \\
\hline Digestibilité MS in vitro (\%) & $\begin{array}{l}1 \\
2\end{array}$ & $\begin{array}{l}70,6 \\
77,8\end{array}$ & $\begin{array}{l}70,1 \\
77,6\end{array}$ & $\begin{array}{l}72,0 \\
79,8\end{array}$ & $\begin{array}{l}73,8 \\
79,8\end{array}$ \\
\hline Gain quotidien moyen $(\mathrm{kg} / \mathrm{j})$ & $\begin{array}{l}1 \mathrm{M} \\
2 \stackrel{M}{2} \\
2 \mathrm{C}\end{array}$ & $\begin{array}{l}1,26 \pm 0,11 \\
1,27 \pm 0,33 \\
1,28 \pm 0,10\end{array}$ & $\begin{array}{l}1,32 \pm 0,20 \\
1,63 \pm 0,17 \\
1,53 \pm 0,12\end{array}$ & $\begin{array}{l}1,31 \pm 0,10 \\
1,48 \pm 0,06 \\
1,24 \pm 0,12\end{array}$ & $\begin{array}{l}1,42 \pm 0,06 \\
1,57 \pm 0,21 \\
1,25 \pm 0,12\end{array}$ \\
\hline Indice de consommation $(\mathrm{kg} / \mathrm{kg})$ & $\begin{array}{ll}1 & M \\
2 & M \\
2 & C\end{array}$ & $\begin{array}{l}6,61 \pm 0,72 \\
6,94 \pm 2,07 \\
6,23 \pm 0,84\end{array}$ & $\begin{array}{l}6,44 \pm 0,73 \\
5,60 \pm 0,62 \\
5,35 \pm 0,44\end{array}$ & $\begin{array}{l}6,22 \pm 0,51 \\
5,80 \pm 0,82 \\
6,42 \pm 0,56\end{array}$ & $\begin{array}{l}5,64 \pm 0,51 \\
5,45 \pm 0,27 \\
6,31 \pm 0,68\end{array}$ \\
\hline Rendement d'abattage (\%) & $\begin{array}{ll}1 & M \\
2 & M \\
2 & C\end{array}$ & $\begin{array}{l}57,4 \pm 1,1 \\
57,1 \pm 0,5 \\
65,3 \pm 0,3\end{array}$ & $\begin{array}{l}57,4 \pm 1,5 \\
57,2 \pm 1,1 \\
65,2 \pm 0,5\end{array}$ & $\begin{array}{l}57,6 \pm 0,4 \\
57,7 \pm 0,9 \\
64,7 \pm 1,1\end{array}$ & $\begin{array}{l}56,1 \pm 3,6 \\
59,9 \pm 1,0 \\
63,4 \pm 1,5\end{array}$ \\
\hline
\end{tabular}

A = Année ( 1 pour la $1^{\text {re }}$ année et 2 pour la $2^{\mathrm{e}}$ année); $T$ = type de taureau ( $M$ pour mixte et $C$ pour culard).

sur les gains de poids et les consommations. Néanmoins, les gains les plus élevés ont été observés avec les variétés Lixis en $1^{\text {re }}$ année et Gracia en $2^{\theta}$ année (tableau I). Les gains de poids dans la présente expérience, avec un apport de $2 / 3$ de la MS sous forme de maïs, sont comparables aux résultats obtenus par Cottyn et al (1984) avec environ $60 \%$, et par Istasse et al (1989) avec $50 \%$. Les indices de consommation ont été les meilleurs avec la variété Lixis chez les taureaux de type mixte et avec la variété Gracia chez les taureaux de type culard. À l'abattage, il n'y a eu que peu d'effet du tỵpe de maïs sur la freinte, le rendement et la composition de la carcasse. En $1^{\text {re }}$ année, les performances et les digestibilités les plus extrêmes ont été observées avec les variétés Ascot et Lixis. Chez les animaux de type mixte, l'augmentation des gains de poids en $2^{\ominus}$ année peut être associée à une proportion d'épis et une teneur en MS plus élévées ainsi qu'à une meilleure digestibilité de la MS.

Remerciements - Ce travail a été réalisé sous les auspices de I'IRSIA, rue de Crayer, 6 , Bruxelles.

Cottyn BG, Boucquè CV, Fiems LO, Buysse FX (1984) Rev Agric 37, 3, 649-660

Gielen $M$, Istasse $L$, Bienfait JM, Legros $P$ (1988) Elev Belg 1, 37-39

Istasse L, Van Eenaeme C, Baldwin P, Khameni Djiele N, Bienfait JM (1989) Ann Méd Vét 133, 39-44 